\title{
The capacity of the human iliotibial band to store elastic energy during running
}

\section{Citation}

Eng, Carolyn M., Allison S. Arnold, Daniel E. Lieberman, and Andrew A. Biewener. 2015. "The Capacity of the Human Iliotibial Band to Store Elastic Energy During Running." Journal of Biomechanics 48 (12) (September): 3341-3348. doi:10.1016/j.jbiomech.2015.06.017.

\section{Published Version}

doi:10.1016/j.jbiomech.2015.06.017

\section{Permanent link}

http://nrs.harvard.edu/urn-3:HUL.InstRepos:33085964

\section{Terms of Use}

This article was downloaded from Harvard University's DASH repository, and is made available under the terms and conditions applicable to Open Access Policy Articles, as set forth at http:// nrs.harvard.edu/urn-3:HUL.InstRepos:dash.current.terms-of-use\#OAP

\section{Share Your Story}

The Harvard community has made this article openly available.

Please share how this access benefits you. Submit a story.

\section{Accessibility}


2 The capacity of the human iliotibial band to store elastic energy during running

7 Carolyn M. Eng ${ }^{1,2}$, Allison S. Arnold ${ }^{1}$, Daniel E. Lieberman ${ }^{2}$, Andrew A. Biewener $^{1}$ ${ }^{1}$ Department of Organismic and Evolutionary Biology, Harvard University,

${ }^{2}$ Department of Human Evolutionary Biology, Harvard University, Cambridge, MA

Journal of Biomechanics Keywords: Elastic energy storage, iliotibial band, fascia, musculoskeletal modeling

17 Correspondence to: Carolyn M. Eng, Department of Ecology and Evolutionary Biology, 18 Brown University, PO Box G-B204, Providence, RI 02912. E-mail:

19 carolyn eng@brown.edu. 


\section{Abstract}

The human iliotibial band (ITB) is a poorly understood fascial structure that may

24 contribute to energy savings during locomotion. This study evaluated the capacity of the

25 ITB to store and release elastic energy during running, at speeds ranging from $2-5 \mathrm{~m} / \mathrm{s}$,

26 using a model that characterizes the three-dimensional musculoskeletal geometry of the

27 human lower limb and the force-length properties of the ITB, tensor fascia lata (TFL),

28 and gluteus maximus (GMax). The model was based on detailed analyses of muscle

29 architecture, dissections of 3-D anatomy, and measurements of the muscles' moment

30 arms about the hip and knee in five cadaveric specimens. The model was used, in

31 combination with measured joint kinematics and published EMG recordings, to estimate

32 the forces and corresponding strains in the ITB during running. We found that forces

33 generated by TFL and GMax during running stretch the ITB substantially, resulting in

34 energy storage. Anterior and posterior regions of the ITB muscle-tendon units (MTUs)

35 show distinct length change patterns, in part due to different moment arms at the hip

36 and knee. The posterior ITB MTU likely stores more energy than the anterior ITB MTU

37 because it transmits larger muscle forces. We estimate that the ITB stores about $1 \mathrm{~J}$ of

38 energy per stride during slow running and $7 \mathrm{~J}$ during fast running, which represents

39 approximately $14 \%$ of the energy stored in the Achilles tendon at a comparable speed.

40 This previously unrecognized mechanism for storing elastic energy may be an

41 adaptation to increase human locomotor economy. 


\section{Introduction}

Because bipedalism is a fundamental derived feature of hominins (species more closely related to humans than chimpanzees), many distinctive features of the human spine and lower extremity are adaptations to improve bipedal locomotor performance.

47 Many adaptations for standing and walking, for example, appear early in hominin evolution including a inferiorly-oriented foramen magnum, a lordotic lumbar spine, and a sagittally-oriented ilium (see Aiello and Dean, 1990; Zollikofer et al., 2005). Additional features that first appear later in the genus Homo may reflect selection for endurance

51 running, including a stabilized sacroiliac joint, an expanded attachment of gluteus

52 maximus, and shorter toes (Bramble and Lieberman, 2004; Lieberman et al., 2006;

53 Rolian et al., 2009). Although the selective factors underlying the evolution of both 54 walking and running are debated, it is likely that locomotor economy played a key role.

55 Hypothesized energy-saving features for walking include long legs and dorsally oriented 56 ischia (Crompton et al., 1998; Pontzer et al., 2009; Robinson, 1972; Sockol et al., 2007).

57 Energy saving features for running in the genus Homo include a long, compliant Achilles

58 tendon and a spring-like median longitudinal arch, which are known to store and recover

59 elastic energy during running in other vertebrates (Biewener, 2003; Ker et al., 1987;

60 Roberts, 2002). In addition, the human lower extremity has a number of fascial

61 structures with elastic properties that are not present in apes, but whether these

62 structures store energy or serve another function remains poorly understood.

63 One of the most interesting of these structures is the iliotibial band (ITB). The ITB

64 is a thickening of the lateral fascia of the thigh that originates on the pelvis and inserts

65 on the tibia; it receives muscle fibers from the tensor fascia lata (TFL) anteriorly and 
66 from the gluteus maximus (GMax) posteriorly (Gottschalk et al., 1989; Gray et al., 1995;

67 Kaplan, 1958; Ober, 1936; Stern, 1972). The ITB is traditionally considered to function

68 as a "strut" during walking, stabilizing the hip in the frontal plane (Gottschalk et al.,

69 1989; Inman, 1947; Kaplan, 1958). However, the high compliance of the ITB (Butler et

70 al., 1984; Derwin et al., 2008; Gratz, 1931), the fact that it crosses both the hip and

71 knee, and the presence of in-series muscles suggest that the ITB may play other roles.

72 If the ITB stretches substantially while transmitting muscle forces, storing elastic energy,

73 then it may decrease the metabolic cost of locomotion. Prior studies have estimated that

74 energy recovered from the Achilles tendon during running reduces muscle work by as

75 much as 35\% (Alexander and Bennet-Clark, 1977; Ker et al., 1987). Whether the ITB

76 also stores and recovers elastic energy, and how this compares to Achilles tendon

77 energy recovery, is unknown.

As a first step toward evaluating the ITB's role in locomotor economy, this study examined the capacity of the ITB to store elastic energy at running speeds ranging from

802 to $5 \mathrm{~m} / \mathrm{s}$. We hypothesized that forces generated by TFL and GMax stretch the ITB

81 during running, storing elastic energy that may be recovered later in the stride. We

82 tested this hypothesis by developing a musculoskeletal model of the ITB and inserting

83 muscles. Our model characterizes the 3-D skeletal geometry, the hip and knee

84 kinematics, and the attachments and force-length (F-L) properties of the ITB, TFL and

85 GMax for an average-sized adult male (femur length: $39.8 \mathrm{~cm}$; tibia length: $36.2 \mathrm{~cm}$ ).

86 Because existing representations of TFL and GMax were not sufficiently accurate for

87 this study, we performed detailed analyses of these muscles' architecture and

88 measured their moment arms (MAs) about the hip and knee in cadaveric specimens. 
89 The TFL has largely been neglected in previous studies of muscle architecture (e.g.,

90 Ward et al., 2009; Wickiewicz et al., 1983) and locomotor function (e.g., Dorn et al.,

91 2012; Sasaki and Neptune, 2006), despite being active during running (Andersson et

92 al., 1997; Mann et al., 1986; Montgomery et al., 1994; Paré et al., 1981). GMax is

93 routinely modeled as a uniarticular hip extensor that inserts on the femur (e.g., Arnold et

94 al., 2010; Delp et al., 1990), despite evidence that a substantial portion of GMax inserts

95 on the ITB (Gray et al., 1995; Stern, 1972). Our refined musculoskeletal model, which

96 addresses these limitations, is available on SimTK (simtk.org). Using this model, we

97 estimated the forces transmitted to anterior and posterior regions of the ITB at body

98 positions corresponding to running, predicted the length changes of each region, and

99 calculated the corresponding ITB strain energies over the course of a stride based on

100 published measurements of the tissue's elastic modulus (Butler et al., 1984; Derwin et

101 al., 2008).

102

103 Materials and methods

104 Muscle architecture measurements

105 We characterized the isometric force-generating capacity of TFL and GMax

106 based on measurements of muscle architecture in three formalin-fixed human cadavers

107 (Table 1). Specimens were dissected and the muscles isolated and removed. Total

108 mass $(M)$ of each muscle was measured; in addition, the masses of four regions of the

109 GMax were measured separately. A muscle fascicle was carefully dissected from each

110 region of GMax and from two regions of TFL and the fascicle lengths $\left(L_{f}\right)$ measured.

111 Surface pennation angles between the fascicles and ITB were also measured. Under 
112 magnification, muscle fiber bundles were isolated from each fascicle and mounted on

113 slides. Following Lieber et al. (1990), bundle sarcomere length $\left(L_{s}{ }^{\prime}\right)$ was determined by

114 laser diffraction and used to calculate optimal fascicle length $\left(L_{t}\right)$ :

$$
L_{f}=L_{f}{ }^{\prime}\left(\frac{2.7 \mu m}{L_{s}^{\prime}}\right)
$$

116 where $2.7 \mu \mathrm{m}$ is the optimal sarcomere length for human muscle (Lieber et al., 1994).

117 Physiological cross-sectional area (PCSA) was calculated from muscle mass and

118 optimal fascicle length (Powell et al., 1984):

$$
P C S A=\frac{M}{\rho \cdot L_{f}}
$$

120 where $\rho$ is muscle density $\left(1.056 \mathrm{~g} / \mathrm{cm}^{3}\right.$; Mendez and Keyes, 1960). Our architecture

121 data for GMax are consistent with data reported by Ward et al. (2009).

122 Muscle moment arm measurements

123 We measured MAs of the muscle-ITB paths in five fresh frozen cadaveric hemi-

124 pelvises obtained from MedCure (Portland, OR). MAs were determined for hip

125 flex/extension, rotation, ab/adduction, and knee flex/extension using the tendon

126 excursion method (An et al., 1984; Brand et al., 1975). We approximated TFL with two

127 Kevlar thread paths (Figure 1A\&B) and GMax with four paths (Figure $1 \mathrm{~A} \& \mathrm{C}$ ). The ITB

128 was left intact during these measurements. Each thread was anchored to a screw eye

129 at the path's insertion, routed over the ITB through plastic tubing to a screw eye at the

130 path's origin, and attached to one of two cable-extension position transducers (PTX101,

131 Celesco, Canoga Park, CA) that measured length changes with an accuracy of \pm 0.32

$132 \mathrm{~mm}$ while applying a tension of 1.4 or $2.8 \mathrm{~N}$. The tubing ensured a repeatable path 
133 along the surface of the ITB and decreased friction. Detailed procedures for defining

134 each path are described in supplementary materials.

$135 \quad$ Hip and knee angles were measured simultaneously with muscle-ITB length

136 changes using a motion tracking system (Polhemus Fastrak, Colchester, VT) and

137 custom software (LabView, National Instruments Corporation, Austin, TX). Receivers

138 were rigidly attached to the pelvis, femur, and tibia to track the segments' positions and

139 orientations. Segment coordinate systems were defined along anatomical axes by

140 digitizing bony landmarks and determining the hip center (Figure S1), as described in

141 the supplementary materials. For each muscle-ITB path, we digitized the origin,

142 insertion and key "via" points that constrained the path with hip or knee motion. We also

143 tracked the relative motions of nine marker pairs sutured along the ITB using high-

144 speed video. These data guided development of the model and were analyzed to

145 determine the hip and knee angles at which the anterior and posterior ITB began to 146 stretch.

147 Each specimen was mounted in a custom frame (Figure 2) that allowed

148 independent control of hip flex/extension, rotation, ab/adduction, and knee flexion

149 following Arnold et al. (2000). Alignment and mounting of the specimen comprised four

150 main steps, each performed with real-time feedback to ensure that the pelvis, femur,

151 and tibia were secured to within $5 \mathrm{~mm}$ and $2^{\circ}$ of the desired alignment. First, the pelvis

152 was secured to a table and aligned with either its medial-lateral axis (for flex/extension

153 MAs) or anterior-posterior axis (for ab/adduction MAs) perpendicular to the table.

154 Second, the femur was mounted on a cart equipped with two concentric rings. The

155 femur was secured to the inner ring so that the femur's long axis (from hip center to the 
156 midpoint between femoral epicondyles) was centered perpendicular to the plane of the 157 rings. Third, the base of the cart was adjusted so that its wheels rolled in an arc about 158 the specimen's hip center. Fourth, the tibia was secured to a locking hinge attached to 159 the inner ring. When measuring knee MAs, the hinge was removed and the tibia was 160 flexed and extended. When measuring hip rotation MAs, the inner ring was rotated 161 relative to the outer ring, which internally and externally rotated the hip. When 162 measuring hip flex/extension or ab/adduction MAs, the cart was rotated about the 163 specimen's hip center, thereby flex/extending or ab/adducting the hip. When measuring 164 MAs about one axis, the other axes were locked in a neutral position (hip flexion $=0^{\circ}$, 165 hip rotation $=5^{\circ}$, hip adduction $=0^{\circ}$, knee flexion $=0^{\circ}$ ). To verify alignment, we 166 monitored coupling of hip angles and ensured that hip adduction varied $<2^{\circ}$ and hip 167 rotation $<4^{\circ}$ over a $75^{\circ}$ range of flexion. When the specimen was aligned for hip 168 ab/adduction, we ensured that hip flexion varied $<2^{\circ}$ and hip rotation $<4^{\circ}$ over a $50^{\circ}$ 169 range of ab/adduction.

170 To measure muscle-ITB MAs, the excursion of each thread path was recorded 171 while slowly moving the specimen through its ranges of hip and knee motion. Excursion 172 and joint angle data were sampled at $10 \mathrm{~Hz}$ (National Instruments BNC-2090 A/D 173 converter). The lengthening excursion versus joint angle data were fit with a fourth order 174 polynomial, and the derivative of the polynomial was averaged across trials to estimate 175 the MA. A minimum of five trials was collected for each condition.

176 Following MA measurements, muscles were freed, cleaned of fat and connective 177 tissue, and weighed (Table 2). In two specimens, the regions of GMax were carefully 
178 dissected to determine the relative masses of the portions that insert on the ITB versus

179 the femur.

Model of TFL, Gmax, and ITB F-L properties

We modified the paths of TFL and GMax muscle-tendon units (MTUs) in the

182 model reported by Arnold et al. (2010) to match our digitized attachments and MA data

183 (Figure 3). Using SIMM (Software for Interactive Musculoskeletal Modeling, v7.0,

184 MusculoGraphics, Santa Rosa, CA), we initially created two paths for TFL and eight for

185 GMax (four to the ITB and four to the femur). Via points and wrapping surfaces were

186 iteratively adjusted so that the model's paths reproduced the three-dimensional paths

187 digitized during the experiments and the model's MAs matched the experimentally

188 determined MAs. Because the model's MAs are extremely consistent with our

189 experimental data (Figures 4 \& S2), we are confident that the model accurately predicts

190 length changes of these MTUs.

191 To estimate strain in regions of the ITB during running, we created three

192 additional MTUs, representative of the major paths of ITB force transmission

193 determined from our experiments (Figure 3B,C). One path accounts for force

194 transmitted by the anterior ITB when TFL is active (TFL-ITB $\left.{ }_{\text {ant }}\right)$. The other paths account

195 for the cumulative force transmitted by the posterior ITB when superior (GMax1,2-

196 ITB $_{\text {post1 }}$ ) or inferior (GMax3,4-ITB post2) portions of GMax are active. Attachments and via

197 points of each path were iteratively adjusted to yield average MAs of the combined

198 MTUs (Figures 4). This model represents the muscles as independent, proximal-to-

199 distal MTUs, even though the ITB is multi-layered and loaded from different directions,

200 based on detailed dissections and on biaxial testing of goat fascia lata, which showed 
201 that the fascia's material properties are not strongly influenced by its biaxial strain 202 environment (Eng et al., 2014).

203 We used a Hill-type muscle model (Delp et al., 1990; Zajac, 1989) to estimate

204 isometric forces generated by TFL-ITB ${ }_{\text {ant }}$, GMax1,2-ITB post1, and GMax3,4-ITB $_{\text {post2 }}$ at

205 different activation levels. Two parameters, maximum isometric force $\left(F_{\max }\right)$ and optimal

206 fiber length $\left(L_{o p t}\right)$, scaled normalized active and passive F-L curves to each muscle

207 (Table S1). $F_{\max }$ and an additional parameter, tendon slack length $\left(L_{T S}\right)$, scaled a

208 normalized "tendon" F-L curve to each MTU. We specified parameters for each MTU

209 based on our architecture measurements and data reported by Ward et al. (2009). We

210 adjusted $L_{T S}$ such that ITB ant and ITB post began to stretch passively at hip and knee

211 angles consistent with our experimental data.

212 For each MTU, we created a normalized F-L curve for the ITB (Figure 5) based

213 on published material properties of the human ITB (Butler et al., 1984; Derwin et al.,

214 2008). We assumed a transition strain of $4 \%$ based on data from goat fascia lata (Eng

215 et al., 2014). Above 4\% strain, we assumed a linear relationship between force and

216 strain with a normalized stiffness $(\tilde{k})$ determined using the ITB's elastic modulus $(E)$,

217 the muscle's $F_{\max }$, and the ITB's effective cross-sectional area (a):

$$
\tilde{k}=\frac{E \times a}{F_{\max }}
$$

The effective cross-sectional area of the ITB was calculated from measurements

220 of thickness and width in cadaveric specimens (see Table S2). The width of each ITB

221 region was measured while placing tension on the inserting muscle and visually

222 assessing ITB strain. We used an elastic modulus of $400 \mathrm{MPa}$, which is consistent with

223 values reported in the literature (Butler et al., 1984; Derwin et al., 2008; Hammer et al., 
224 2012; Steinke et al., 2012). Below 4\% strain, in the toe region, we decreased stiffness

225 by a factor of $2 / 3$. At $F_{\max }$, the ITB strains $5-11 \%$ in our model, which seems plausible

226 given the range of yield strains reported in the literature (10-27\%; (Butler et al., 1984;

227 Hammer et al., 2012; Hinton et al., 1992). The regional variation in strain at $F_{\max }$ is

228 consistent with our measurements of ITB thickness, which are relatively uniform in

229 anterior and posterior regions despite the fact that the inserting muscles differ

230 substantially in force-generating capacity.

231 Assessment of ITB energy storage

232 We used our model in combination with published joint kinematics and EMG

233 recordings to examine the capacity of the ITB to store elastic energy during running.

234 First, we calculated the lengths of the MTUs at hip and knee angles corresponding to

235 running using data from 10 experienced runners, at speeds of $2,3,4$, and $5 \mathrm{~m} / \mathrm{s}$

236 (Hamner and Delp, 2013). Next, we divided MTU lengths into muscle fiber lengths and

237 ITB lengths by independently activating each MTU in the model and solving for the

238 lengths at which the muscle and ITB forces were equivalent, accounting for pennation

239 angle. Maximum activation levels for running were assumed to range between $20 \%$ and

$24065 \%$ of the EMG activation measured during a maximum voluntary contraction (MVC).

241 In particular, we set each muscle's maximum activation to $20 \%, 35 \%, 50 \%$, or $65 \%$ to

242 estimate ITB strains during running at 2, 3, 4, and $5 \mathrm{~m} / \mathrm{s}$, respectively. These values are

243 based on reported maximum activations of $20 \%$ MVC in GMax and TFL during level

244 walking (Perry, 1992), 40\% MVC in GMax and TFL during level running at $4.0 \mathrm{~m} / \mathrm{s}$

245 (Montgomery et al., 1994), and 65\% MVC in GMax during level running at $4.5 \mathrm{~m} / \mathrm{s}$

246 (Swanson and Caldwell, 2000). Time-varying patterns of activity were derived from 
247 EMG recordings reported for GMax and TFL (Jönhagen et al., 1996; Montgomery et al.,

248 1994; Paré et al., 1981; Swanson and Caldwell, 2000), which we scaled to the

249 maximum activation at each speed (Figure 7). Lastly, we estimated energy storage

250 capacity at each speed by integrating the ITB F-L curves from $L_{T S}$ to peak ITB length

251 during running. Length changes of the ITB were determined relative to slack length.

252 Total elastic energy stored in the posterior ITB was calculated as the sum of the

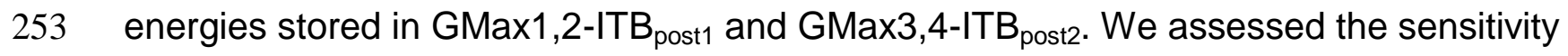

254 of our analysis to the F-L properties by varying normalized stiffness by $\pm 20 \%$ and

255 transition strain by $\pm 2 \%$ (Figure 5) and re-calculating energy storage.

257 Results

The TFL and GMax MTUs in our model undergo substantial excursions during 259 running (Figure 6). Because of its hip flexion and knee extension MAs, TFL-ITB ant is 260 maximally stretched during early swing, when the hip is extended and the knee flexed

261 (Figure 3C). EMG recordings show that TFL is highly activated during this time (Figures $2626 \&$ 7) (Montgomery et al., 1994; Paré et al., 1981). In contrast, because of their role in 263 hip extension and knee flexion MAs, GMax-ITB post1 and GMax-ITB $\mathrm{Bost2}_{2}$ are maximally 264 stretched during late swing, when the hip flexes and the knee extends (Figure 3C).

265 EMG recordings show that GMax is highly activated during this time (Figures 6 \& 7)

266 (Jönhagen et al., 1996; Swanson and Caldwell, 2000). Inferior portions of GMax

267 lengthen about 7\% more than proximal portions, due to larger hip extension MAs when 268 the hip is flexed. 
The largest strains in ITB ant occur in early swing (Figure 7A), with ITB ant stretching

$270 \quad 0.9$ to $1.7 \mathrm{~cm}$ beyond slack length in our model. TFL muscle fiber length is longer than

271 optimal when it begins generating force in late stance, and near optimal when it is

272 maximally activated in early swing. Peak strains in ITB post occur in late swing (Figure

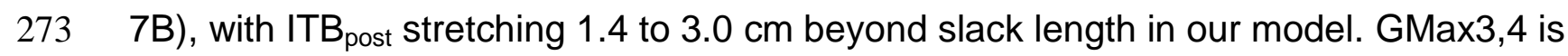

274 shorter than optimal length when it begins generating force in mid swing; however, it is

275 stretched beyond optimal length as the hip flexes in swing. In late swing, when GMax3,4

276 is maximally activated, it operates near optimal length and generates forces that stretch

277 ITB $_{\text {post }}$ in our model. A similar pattern occurs in GMax1,2-ITB post2. Passive strains in the

278 ITB, without muscle activation, are relatively small in our model. ITB ant strains $1.7 \%$ and

$279 \quad$ ITB $_{\text {post }}$ strains $1.1 \%$ over the stride cycle when the muscles are not activated.

280 Because the TFL and GMax MTUs are stretched to relatively long lengths when

281 the muscles are active during running, both anterior and posterior regions of the ITB in

282 our model have the capacity to store elastic energy. We estimate that ITB $\mathrm{ant}_{\text {strains }}$

283 about $4 \%$ during running at $5 \mathrm{~m} / \mathrm{s}$, which means that the ITBant may store nearly $1 \mathrm{~J}$ of

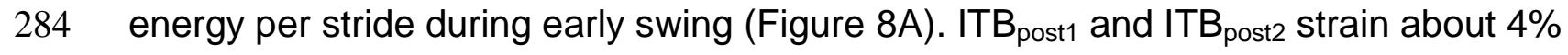

285 during slow running and 7\% during faster running in late swing when GMax is maximally

286 active in our model. These data suggest that the ITB post may store as much as $6 \mathrm{~J}$ per

287 stride during late swing (Figure 8B).

289 Discussion

290 This is the first study to quantitatively characterize the 3-D musculoskeletal

291 geometry of the human ITB and its inserting muscles. Dissections confirmed that all 
292 fibers of TFL insert into the anterior ITB and a large fraction of GMax (40-70\% by mass)

293 inserts into the posterior ITB. Thus, the ITB likely transmits substantial force.

294 Additionally, our MA measurements confirmed that the inserting muscles have relatively

295 large MAs about the hip, and thus undergo large MTU excursions, with hip flexion and

296 extension. In combination, the ITB's high compliance and its potential to transmit force

297 while changing length, suggest a plausible, previously unrecognized mechanism for

298 storing elastic energy during running.

299 We created a model that characterizes the geometry and F-L properties of the

300 ITB, TFL, and GMax to test the hypothesis that forces generated by TFL and GMax

301 stretch the ITB during running, storing elastic energy. Analysis of the model revealed

302 that the ITB has the capacity to store $7 \mathrm{~J}$ per stride during running at $5 \mathrm{~m} / \mathrm{s}$. The

303 posterior ITB stores substantially more energy than the anterior ITB because it transmits

304 larger muscle forces.

305 How does the amount of energy stored in the ITB during running compare to 306 energy stored in the Achilles tendon? Using a 3-D model and static optimization, Lai et 307 al. (2014) estimated that the soleus and gastrocnemius store 40-50 J per stride in the 308 Achilles tendon at slow to fast running speeds $(3.5-5 \mathrm{~m} / \mathrm{s})$, an estimate consistent with 309 previous experimental studies (Alexander and Bennet-Clark, 1977; Hof et al., 2002; Ker 310 et al., 1987). We therefore calculate that the combined anterior and posterior ITB stores $31114 \%$ as much energy as the Achilles tendon at a $5 \mathrm{~m} / \mathrm{s}$ pace.

312 To provide additional context, we compared energy stored in the ITB to hip

313 muscle work during running. Sasaki and Neptune (2006) used a muscle-driven dynamic 314 simulation to estimate the mechanical work performed by hip muscles and series elastic 
315 elements during running at $2.4 \mathrm{~m} / \mathrm{s}$. They reported that the hip extensors do $40 \mathrm{~J}$ of

316 work per stride during stance, while the hip flexors do $6 \mathrm{~J}$ of work during swing.

317 Recovery of $2 \mathrm{~J}$ from ITB post during slow running could account for $5 \%$ of the work done

318 by hip extensors in stance, while recovery of $0.3 \mathrm{~J}$ from ITB ant could contribute $5 \%$ of the

319 work done by hip flexors in swing. Although the extent to which energy recovery would

320 drive selection for endurance running is unknown, these comparisons suggest that

321 energy storage in the ITB is not negligible.

322 This analysis has several limitations. First, although our data confirm that forces

323 generated by TFL and GMax stretch the ITB during running, storing useful energy, our

324 study did not test whether the human ITB reduces muscle work or enhances locomotor

325 economy. Second, uncertainty exists in the parameters used to derive the F-L curves.

326 For example, our measures of ITB width and thickness in cadaveric specimens may not

327 be representative of healthy young subjects, thus potentially underestimating ITB

328 stiffness. However, varying normalized stiffness by $\pm 20 \%$ and transition strain by $\pm 2 \%$

329 altered our estimates of energy storage by only $0.1 \mathrm{~J}$ in the anterior ITB and by $1.2 \mathrm{~J}$ in

330 the posterior ITB at the fastest running speed. At the slowest running speed, varying

331 stiffness and transition strain altered our estimates of energy storage by about $0.05 \mathrm{~J}$.

332 Thus, we are reasonably confident in our model of the ITB's force-strain behavior and

333 that the ITB contributes to energy storage at all running speeds. Third, we estimated the

334 peak forces generated by TFL and GMax ignoring the muscles' force-velocity (F-V)

335 properties and assuming the muscles' activation patterns during running. If the muscles

336 shorten substantially during running, or if we overestimated activation, then we likely

337 overestimated ITB energy storage. It is plausible, however, that the ITB's length and 
338 compliance allow GMax to operate nearly isometrically when generating maximum force

339 in late stance, mitigating the effects of F-V properties on muscle-ITB mechanics. In the

340 running simulations described by Lai et al. (2014), muscles inserting on the Achilles

341 tendon contracted nearly isometrically across a range of running speeds. Lastly, we

342 estimated the capacity of the ITB to store elastic energy during running but not walking.

343 It is likely that the ITB transmits smaller forces, and thus stores less energy, during

344 walking than reported here.

345 Our study has implications for understanding the evolution of human bipedalism.

346 While these data do not exclude the possibility that the ITB stores substantial energy

347 during walking, selection for the capacity to run long distances would have presented

348 unique demands on the anatomy and physiology of Homo (see Bramble and Lieberman

349 (2004) for review). Among these demands is the need to efficiently accelerate the swing

350 limb, which is long and massive in humans (14\% body mass) compared to chimpanzees

351 (9\% body mass; Zihlman and Brunker, 1979). The human ITB is stretched substantially

352 just before swing, when the TFL is active and the hip is extending (Figures 6 \& 7).

353 Subsequent recoil of the ITB may help accelerate the swing limb. Although the

354 energetic cost of running is primarily determined by muscle forces that support the body

355 during stance (Kram and Taylor, 1990), the cost of accelerating the swing limb may be

356 as much as $27 \%$ of total metabolic cost (Marsh et al., 2004; Modica and Kram, 2005;

357 Myers and Steudel, 1985). Thus, selection for increased running economy may have

358 favored traits that increase swing phase energy recovery in Homo. The need to

359 decrease locomotor costs may also help explain the expansion of GMax evident in

360 Homo. This adaptation is thought to play a role in trunk stabilization during endurance 
361 running (Lieberman et al., 2006), but it may also facilitate elastic energy storage by

362 increasing the forces transmitted to the ITB as it is stretched in late swing.

\section{Acknowledgements}

364 The authors fondly remember Farish A. Jenkins Jr. (1940-2012) for many

365 stimulating and insightful discussions. Professor Jenkins helped guide C.M.E.'s

366 dissertation research, which provided the basis for this study, and he deserves much

367 credit. The authors thank two anonymous reviewers for constructive comments that

368 significantly improved this manuscript. We gratefully acknowledge Delande Justinvil and

369 Zachary Lewis for technical assistance during the moment arm experiments. We thank

370 Casey Boyle and Yasmin Rawlins for assistance during pilot studies, and we thank

371 Andrew Mountcastle and Glenna Clifton for help with videography. We are grateful to

372 Tom Roberts for helpful comments on a preliminary version of the manuscript. This

373 research was funded by a Wenner-Gren Dissertation Fieldwork Grant to C.M.E. under

374 award no. 8588.

375

376

377

378 


\section{Figure legends}

Figure 1. A: Lateral view of the human ITB showing paths of the inserting muscles, TFL and GMax, as characterized during the moment arm measurements. B: Lateral diagram showing the anterior and posterior paths of TFL. C: Posterior diagram showing the superior $(G \max 1,2)$ and inferior $($ GMax3,4) regions of GMax. Muscle-ITB paths are described in the supplementary materials.

Figure 2. Hardware and procedure for measuring hip ab/adduction and rotation moment arms. The hardware consisted of a fixed table for aligning and securing the pelvis, an adjustable cart for moving the femur through a range of hip ab/adduction angles, and a set of concentric rings for rotating the femur about its mechanical axis, following Arnold et al. (2000). Receivers (shown in gray) were rigidly attached to the pelvis, femur, and tibia to track their motions in real time. 1: The pelvis was secured to a fixed table with its anterior-posterior axis perpendicular to the table. 2: The femur was secured to the inner of the two rings so that the femur's long axis was centered perpendicular to the plane of the rings. 3: The bases of the cart were adjusted so that the cart's wheels rolled in an arc about the specimen's hip joint center. 4: The tibia was secured to a locking hinge. Hip flex/extension moment arms were measured by re-orienting the pelvis on the table so that its medial-lateral axis was perpendicular to the table. More details are provided in the supplementary materials.

Figure 3. Lower extremity model modified from Arnold et al. (2010). A: Lateral view showing the two TFL-ITB paths that reproduce our experimental data. B: Posterior view showing the four GMax-ITB paths originating on the ilium, sacrum, and coccyx and inserting on the ITB. C: Lateral view of the combined MTU paths used to estimate energy storage. TFL-ITB ${ }_{\text {ant }}$, GMax1,2-ITB post1 $_{\text {, and }}$ GMax3,4-ITB post2 paths are shown at touchdown, midstance, toeoff, and midswing during running at $5 \mathrm{~m} / \mathrm{s}$. The TFL-ITB ant MTU is maximally stretched in early swing, while the GMax-ITB post $_{\text {MTUs are most }}$ stretched during late swing.

Figure 4. Hip and knee moment arms of TFL-ITB ${ }_{\text {ant }}$ and GMax-ITB $\mathrm{B}_{\text {post }}$ compared with experimental data. A: TFL has a large hip flexion MA that increases as the hip flexes. B: TFL has a large hip abduction MA that increases with hip abduction. C: The most posterior part of TFL has a small knee extension MA that decreases with knee flexion. D,G: All portions of GMax that insert on the ITB have large hip extension MAs. E: The superior portions of GMax have hip abduction MAs. $\mathrm{H}$ : The inferior portions of GMax have hip adduction MAs. F,I: The portions of GMax that insert on the ITB have small knee flexion MAs. Solid lines and shaded regions indicate the means and standard deviations of experimentally determined MAs from five cadaveric limbs. A-C: Dashed lines show the MAs of TFL1-ITB (dark gray), TFL2-ITB (light gray), and the combined TFL1,2-ITB ant (black) predicted by our model. D-I: Dashed lines show the MAs (from superior to inferior) of GMax1 (dark gray), GMax2 (light gray), GMax3 (dark gray), GMax4 (light gray), and the combined paths for GMax1,2-ITB post1 (black, D-F) and GMax3,4-ITB post2 (black, G-I) as predicted by our model. Note the y-axes have different scales. 
Figure 5. Normalized force-length curves for anterior and posterior regions of the ITB derived from experimental data (Butler et al., 1984; Derwin et al., 2008; Eng et al., 2014). Curves are shown for TFL-ITB ant (short dash), GMax1,2-ITB post1 (long dash), and GMax3,4-ITB $B_{\text {post2 }}$ (dot-dash) with shaded regions indicating $\pm 20 \%$ stiffness used in the sensitivity analysis. The ITB is more compliant than tendon (solid line), as shown by the typical tendon force-length curve generated by Millard et al. (2013) and by ultrasoundbased measures of tendon force-length properties reported by Magnusson et al. (2001; light gray). This figure is adapted from figure 3 in Millard et al. (2013).

Figure 6. Length changes of TFL-ITB and GMax-ITB MTUs during fast running $(5 \mathrm{~m} / \mathrm{s})$. TFL-ITB ant (light gray) stretches during stance phase, while GMax1,2-ITB post1 $_{\text {(dark }}$

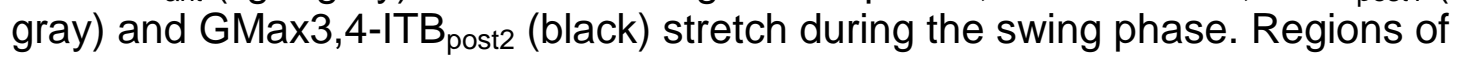
intermediate muscle activity (10-30\% activation; intermediately thickened portion of each curve) and maximal muscle activity (31-65\% activation; thickened portion of each curve) demonstrate that these muscles are maximally active when the MTU is near maximum length.

Figure 7. TFL-ITB ant and GMax3,4-ITB post activation patterns over the stride cycle. For each muscle, the time-varying pattern was scaled to an activation level of $20 \%, 35 \%$, $50 \%$, or $65 \%$ during running at $2,3,4$, and $5 \mathrm{~m} / \mathrm{s}$, respectively (increasingly darker lines). Filled squares on each line indicate toe-off. Superimposed are plots of force versus normalized fiber length and force versus ITB strain at key points in the gait cycle during running at $5 \mathrm{~m} / \mathrm{s}$. Circles on the curves show where the muscle or ITB is acting at that point in the gait cycle. A: TFL is longer than optimal length $\left(L_{o p t}\right)$ prior to toe-off when the muscle begins to generate force. When TFL is maximally activated in early swing, it operates near optimal length and stretches ITB ant to its longest length in our model. B: GMax3,4 is shorter than optimal length when it begins generating force in mid swing and is stretched beyond optimal length in swing. When GMax3,4 is maximally activated in late swing, it operates near optimal length and stretches ITB post to its longest length in our model.

Figure 8. Elastic energy stored in the ITB during running at 2,3 , 4, and $5 \mathrm{~m} / \mathrm{s}$ as

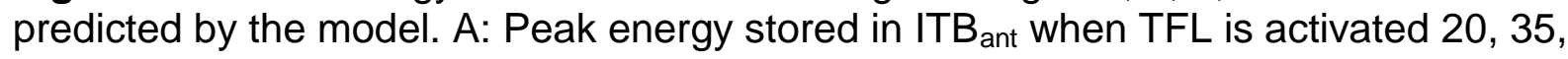
50 , or $65 \%$. B: Peak energy stored in ITB post $_{\text {w }}$ when GMax is activated 20, 35, 50, or $65 \%$. The energy stored in GMax-ITB post is calculated as the sum of energies stored in GMax1,2-ITB post1 $_{\text {(gray) and GMax3,4-ITB }}$ post2 (white). 
Aiello, L.C., Dean, C., 1990. An Introduction to Human Evolutionary Anatomy. Academic Press, Boston.

Alexander, R.M., Bennet-Clark, H.C., 1977. Storage of elastic strain energy in muscle and other tissues. Nature 265, 114-117.

An, K.N., Takahashi, K., Harrigan, T.P., Chao, E.Y., 1984. Determination of muscle orientations and moment arms. Journal of Biomechanical Engineering 106, 280282.

Andersson, E.A., Nilsson, J., Thorstensson, A., 1997. Intramuscular EMG from the hip flexor muscles during human locomotion. Acta Physiologica Scandinavica 161, 361-370.

Arnold, A.S., Salinas, S., Asakawa, D.J., Delp, S.L., 2000. Accuracy of muscle moment arms estimated from MRI-based musculoskeletal models of the lower extremity. Computer Aided Surgery 5, 108-119.

Arnold, E.M., Hamner, S.R., Seth, A., Millard, M., Delp, S.L., 2013. How muscle fiber lengths and velocities affect muscle force generation as humans walk and run at different speeds. Journal of Experimental Biology 216, 2150-2160.

Arnold, E.M., Ward, S.R., Lieber, R.L., Delp, S.L., 2010. A model of the lower limb for analysis of human movement. Annals of Biomedical Engineering 38, 269-279.

Biewener, A.A., 2003. Animal Locomotion. Oxford University Press, Oxford.

Bramble, D., Lieberman, D., 2004. Endurance running and the evolution of Homo. Nature 432, 345-352.

Brand, P.W., Cranor, K.C., Ellis, J.C., 1975. Tendon and pulleys at the metacarpophalangeal joint of a finger. Journal of Bone and Joint Surgery 57, 779-784.

Butler, D.L., Grood, E.S., Noyes, F.R., Zernicke, R.F., Brackett, K., 1984. Effects of structure and strain measurement technique on the material properties of young human tendons and fascia. Journal of Biomechanics 17, 579-596. 
Crompton, R.H., Li, Y., Wang, W., Günther, M.M., Savage, R., 1998. The mechanical effectiveness of erect and "bent-hip, bent-knee" bipedal walking in Australopithecus afarensis. Journal of Human Evolution 35, 55-74.

Delp, S.L., Loan, J.P., Hoy, M.G., Zajac, F.E., Topp, E.L., Rosen, J.M., 1990. An interactive graphics-based model of the lower extremity to study orthopaedic surgical procedures. IEEE Transactions on Biomedical Engineering 37, 757-767.

Derwin, K.A., Baker, A.R., Spragg, R.K., Leigh, D.R., Farhat, W., lannotti, J.P., 2008. Regional variability, processing methods, and biophysical properties of human fascia lata extracellular matrix. Journal of Biomedical Materials Research Part A 84, 500-507.

Dorn, T.W., Schache, A.G., Pandy, M.G., 2012. Muscular strategy shift in human running: dependence of running speed on hip and ankle muscle performance. Journal of Experimental Biology 215, 1944-1956.

Eng, C.M., Pancheri, F.Q., Lieberman, D.E., Biewener, A.A., Dorfmann, L., 2014. Directional differences in the biaxial material properties of fascia lata and the implications for fascia function. Annals of Biomedical Engineering 42, 1224-1237.

Gottschalk, F., Kourosh, S., Leveau, B., 1989. The functional anatomy of tensor fasciae latae and gluteus medius and minimus. Journal of Anatomy 166, 179-189.

Gratz, C.M., 1931. Tensile strength and elasticity tests on human fascia lata. The Journal of Bone and Joint Surgery 13, 334-340.

Gray, H., Williams, P.L., Bannister, L.H., 1995. Gray's Anatomy: The Anatomical Basis of Medicine and Surgery. Churchill Livingstone, Edinburgh.

Hammer, N., Lingslebe, U., Aust, G., Milani, T.L., Hädrich, C., Steinke, H., 2012. Ultimate stress and age-dependent deformation characteristics of the iliotibial tract. Journal of the Mechanical Behavior of Biomedical Materials 16, 81-86.

Hamner, S.R., Delp, S.L., 2013. Muscle contributions to fore-aft and vertical body mass center accelerations over a range of running speeds. Journal of Biomechanics 46, 780-787. 
Hinton, R., Jinnah, R.H., Johnson, C., Warden, K., Clarke, H.J., 1992. A biomechanical analysis of solvent-dehydrated and freeze-dried human fascia lata allografts: A preliminary report. American Journal of Sports Medicine 20, 607-612.

Hof, A., Van Zandwijk, J., Bobbert, M., 2002. Mechanics of human triceps surae muscle in walking, running and jumping. Acta Physiologica Scandinavica 174, 17-30.

Inman, V.T., 1947. Functional aspects of the abductor muscles of the hip. The Journal of Bone and Joint Surgery 29, 607-619.

Jönhagen, S., Ericson, M., Nemeth, G., Eriksson, E., 1996. Amplitude and timing of electromyographic activity during sprinting. Scandinavian Journal of Medicine and Science in Sports 6, 15-21.

Kaplan, E.B., 1958. The iliotibial tract: Clinical and morphological significance. The Journal of Bone and Joint Surgery 40, 817-832.

Ker, R.F., Bennett, M.B., Bibby, S.R., Kester, R.C., Alexander, R.M., 1987. The spring in the arch of the human foot. Nature 325, 147-149.

Kram, R., Taylor, C.R., 1990. Energetics of running: A new perspective. Nature 346, 265-267.

Lai, A., Schache, A.G., Lin, Y.-C., Pandy, M.G., 2014. Tendon elastic strain energy in the human ankle plantar-flexors and its role with increased running speed. Journal of Experimental Biology 217, 3159-3168.

Lieber, R.L., Fazeli, B.M., Botte, M.J., 1990. Architecture of selected wrist flexor and extensor muscles. Journal of Hand Surgery 15A, 244-250.

Lieber, R.L., Loren, G.J., Fridén, J., 1994. In vivo measurement of human wrist extensor muscle sarcomere length changes. Journal of Neurophysiology 71, 874-881.

Lieberman, D.E., Raichlen, D.A., Pontzer, H., Bramble, D.M., Cutright-Smith, E., 2006. The human gluteus maximus and its role in running. Journal of Experimental Biology 209, 2143-2155. 
Magnusson, S.P., Aagaard, P., Rosager, S., Dyre-Poulsen, P., Kjaer, M., 2001.

580 Load-displacement properties of human triceps surae aponeurosis in vivo. Journal of

581 Physiology (London) 531, 277-288.

Mann, R., Moran, G., Dougherty, S., 1986. Comparative electromyography of the lower extremity in jogging, running, and sprinting. American Journal of Sports Medicine $14,501-510$.

Marsh, R.L., Ellerby, D.J., Carr, J.A., Henry, H.T., Buchanan, C.I., 2004. Partitioning the energetics of walking and running: Swinging the limbs is expensive. Science 303, 80-83.

Mendez, J., Keys, A., 1960. Density and composition of mammalian muscle. Metabolism: Clinical and Experimental 9, 184-188.

Millard, M., Uchida, T., Seth, A., Delp, S.L., 2013. Flexing computational muscle: Modeling and simulation of musculotendon dynamics. Journal of Biomechanical Engineering 135, 1-11.

Modica, J.R., Kram, R., 2005. Metabolic energy and muscular activity required for leg swing in running. Journal of Applied Physiology 98, 2126-2131.

Montgomery, W.H., Pink, M., Perry, J., 1994. Electromyographic analysis of hip and knee musculature during running. American Journal of Sports Medicine 22, 272278.

Myers, M., Steudel, K., 1985. Effect of limb mass and its distribution on the energetic cost of running. Journal of Experimental Biology 116, 363-373.

Ober, F.R., 1936. The role of the iliotibial band and fascia lata as a factor in the causation of low-back disabilities and sciatica. The Journal of Bone and Joint Surgery $18,105-110$.

Paré, E., Stern, J., Schwartz, J., 1981. Functional differentiation within the tensor fasciae latae. The Journal of Bone and Joint Surgery 63, 1457-1471.

Perry, J., 1992. Gait Analysis: Normal and Pathological Function. Slack Inc., Thorofare, NJ. 
Pontzer, H., Raichlen, D.A., Sockol, M.D., 2009. The metabolic cost of walking in

Sasaki, K., Neptune, R.R., 2006. Muscle mechanical work and elastic energy utilization during walking and running near the preferred gait transition speed. Gait and Posture 23, 383-390.

of skeletal muscle tension from architectural determinations in guinea pig hindlimbs. Journal of Applied Physiology 57, 1715-1721.

Roberts, T., 2002. The integrated function of muscles and tendons during locomotion. Comparative Biochemistry and Physiology-Part A: Molecular \& Integrative Physiology 133, 1087-1099.

\section{Robinson, J.T., 1972. Early Hominid Posture and Locomotion. University of Chicago Press, Chicago.}

Rolian, C., Lieberman, D.E., Hamill, J., Scott, J.W., Werbel, W., 2009. Walking,
running and the evolution of short toes in humans. Journal of Experimental Biology 212, 713-721.

654 

Mackaye, H., Vignaud, P., Brunet, M., 2005. Virtual reconstruction of Sahelanthropus 

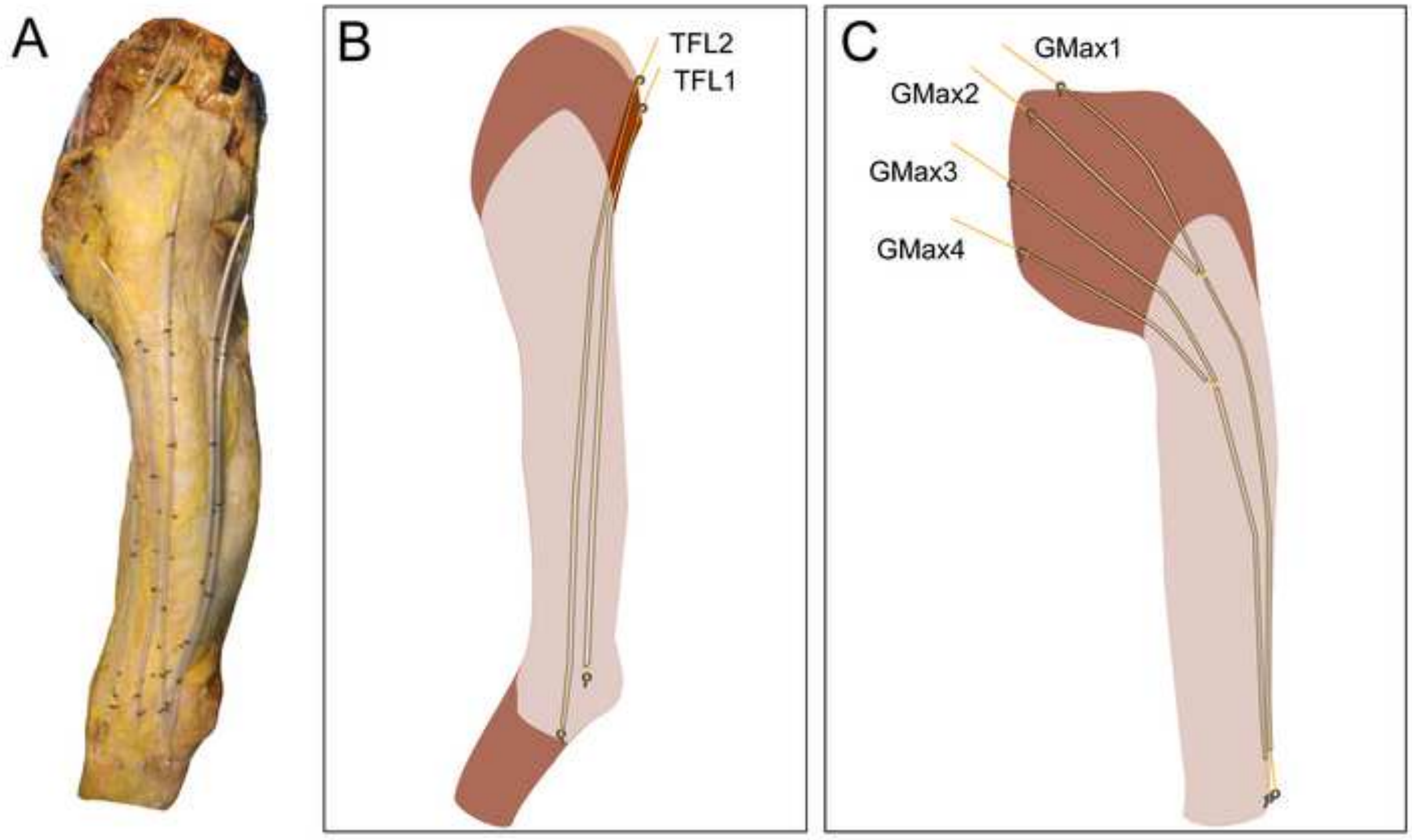


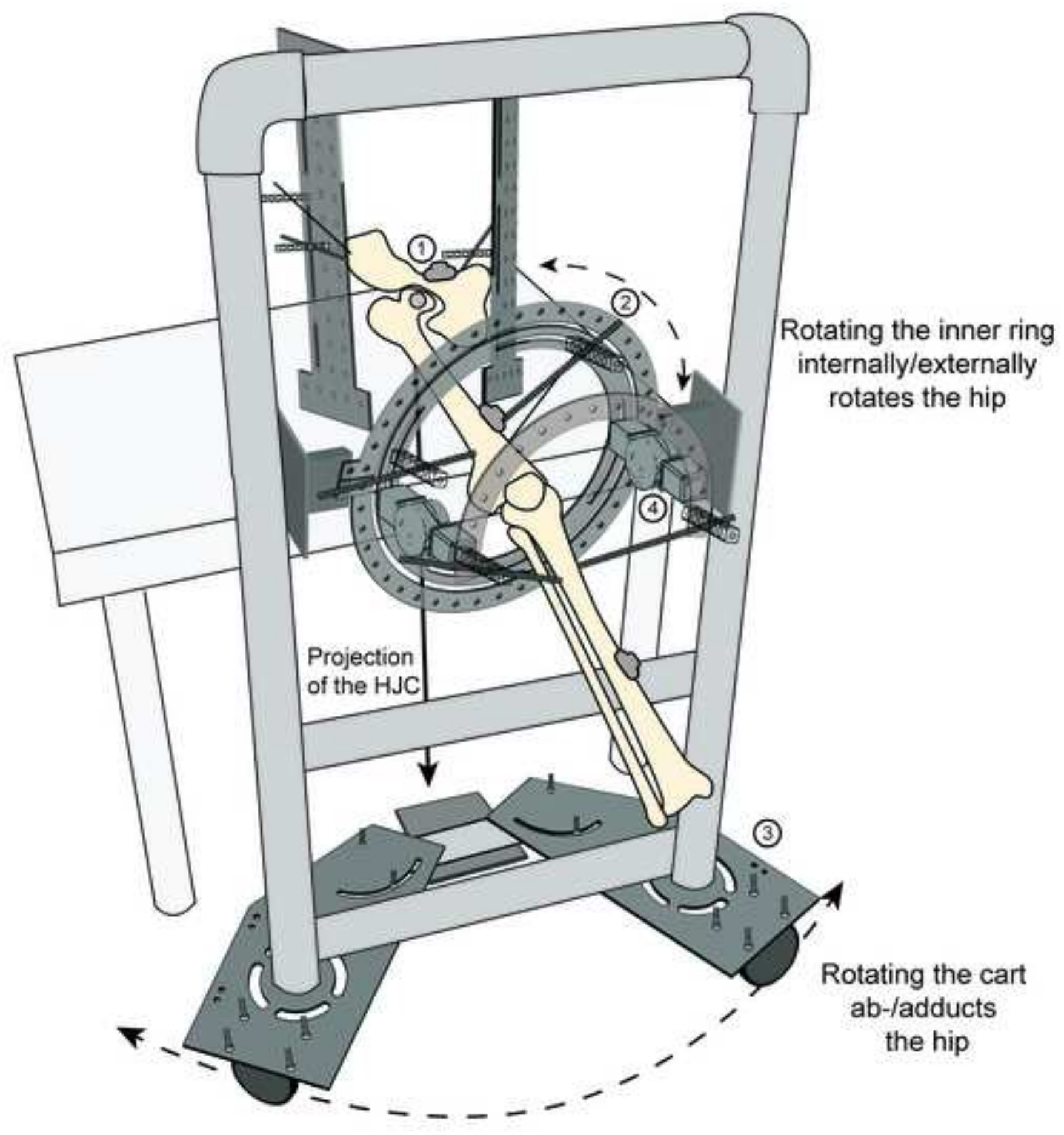


Click here to download high resolution image

A

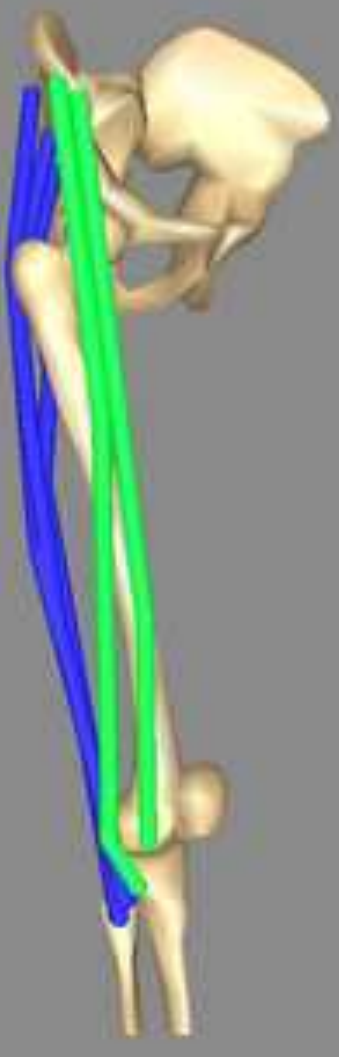

B

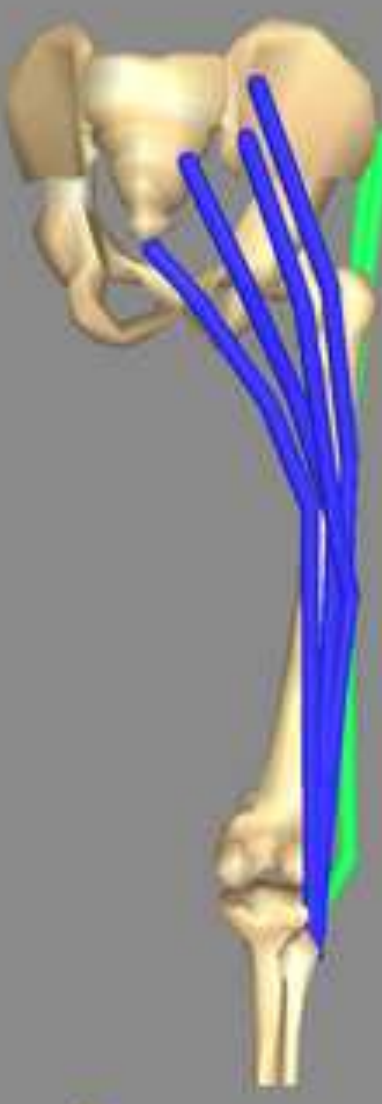

C
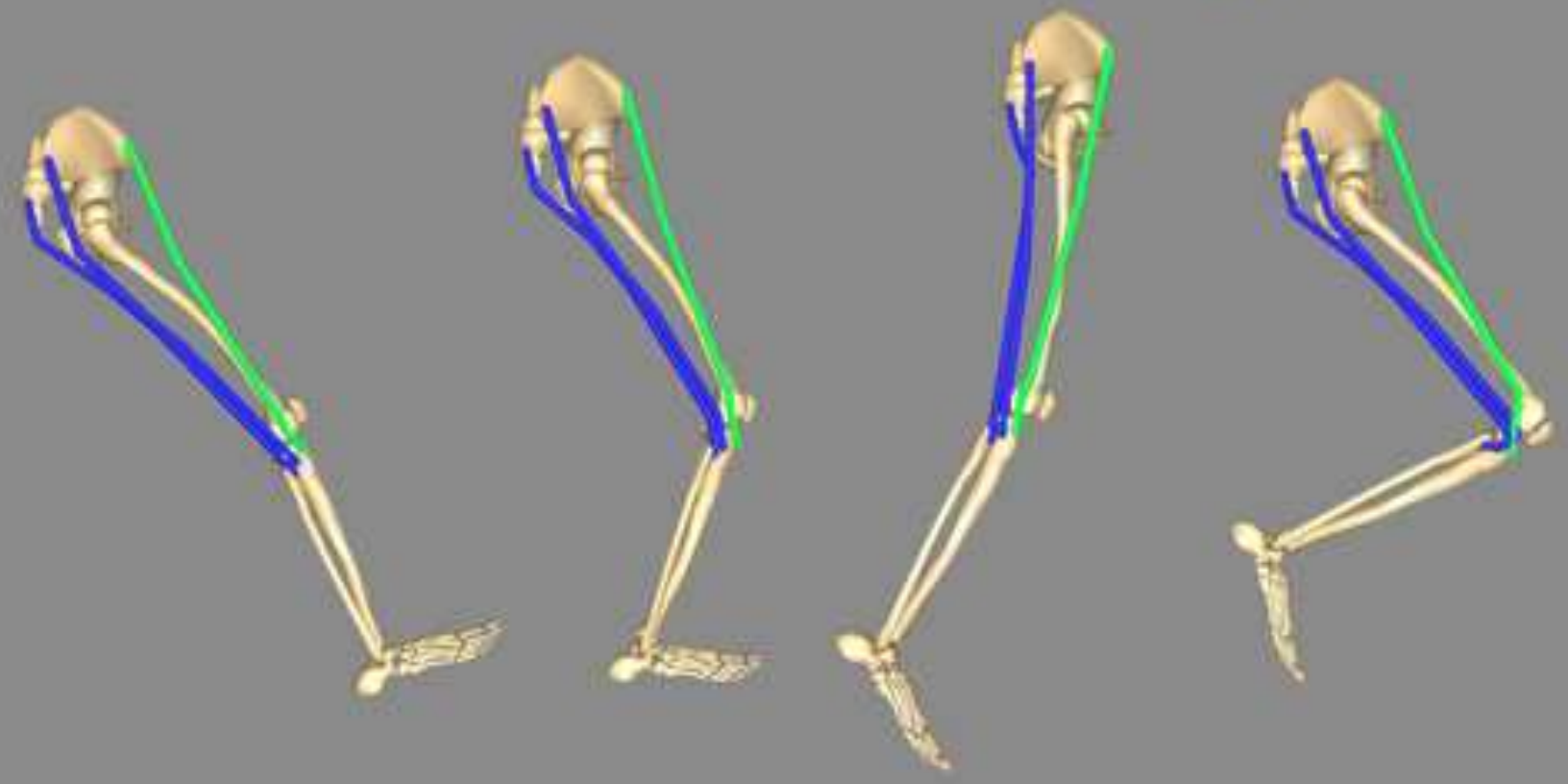

Touchdown

Midstance

Toeoff

Midswing 
A

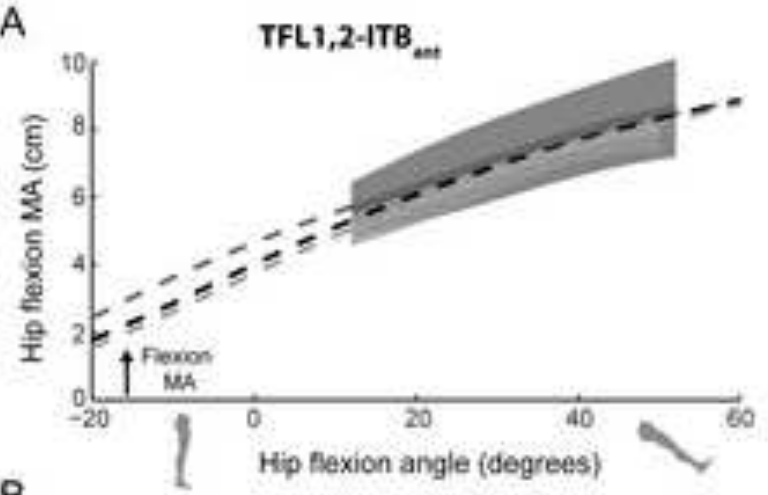

B
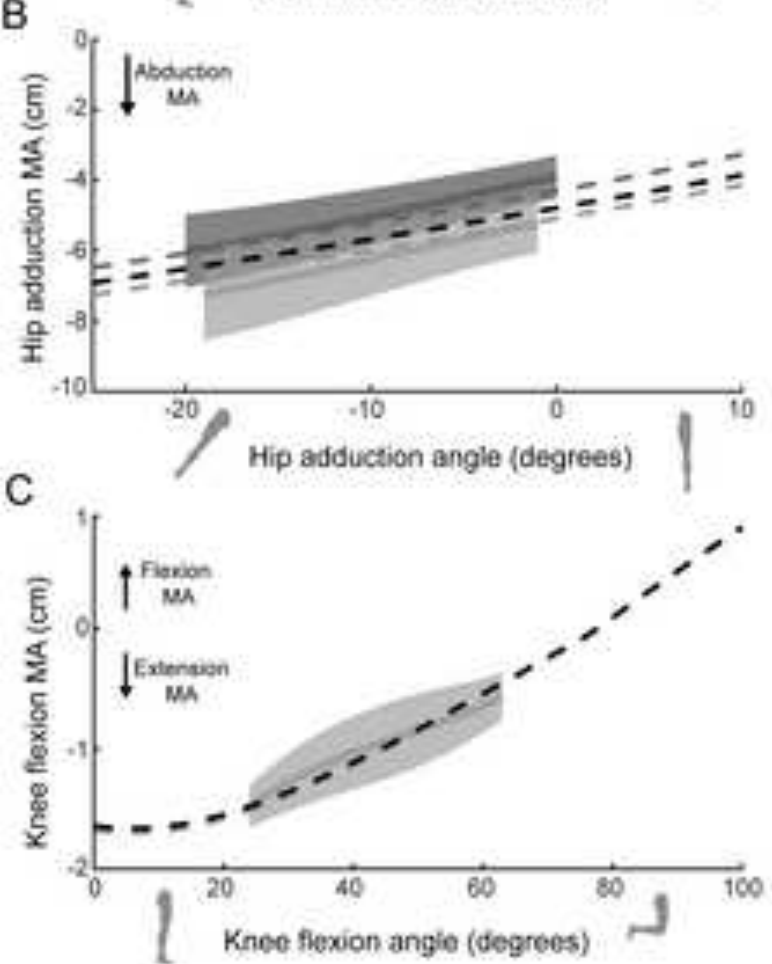

D

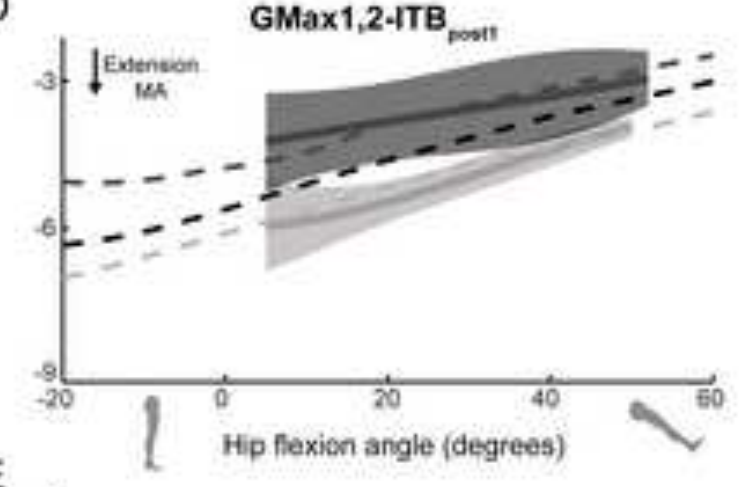

E

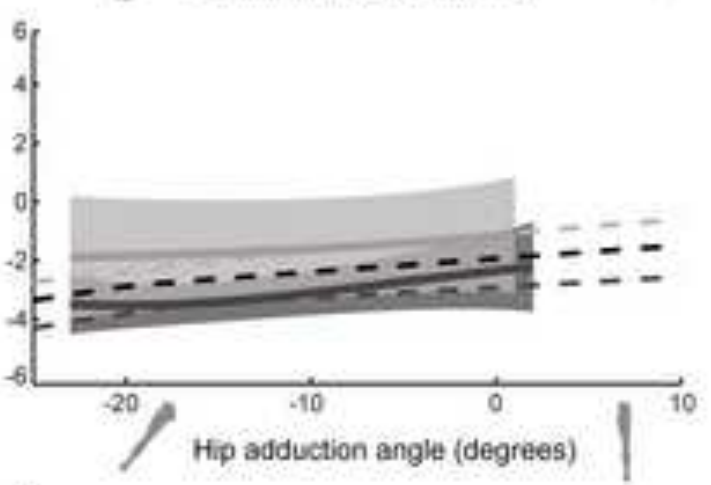

$\mathrm{F}$

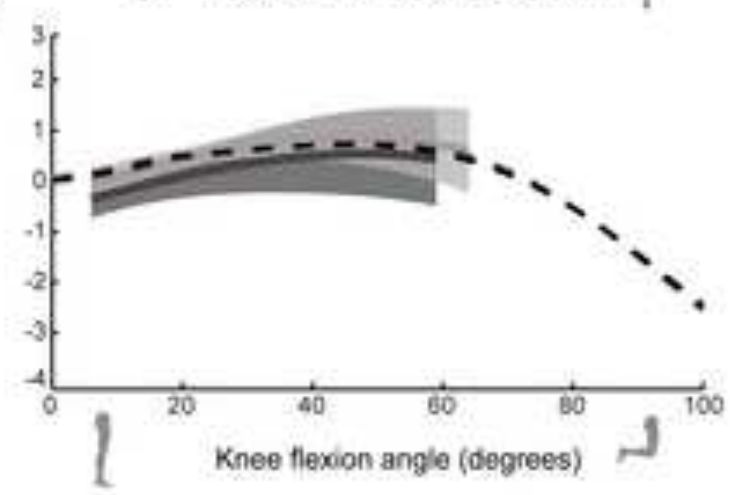

G

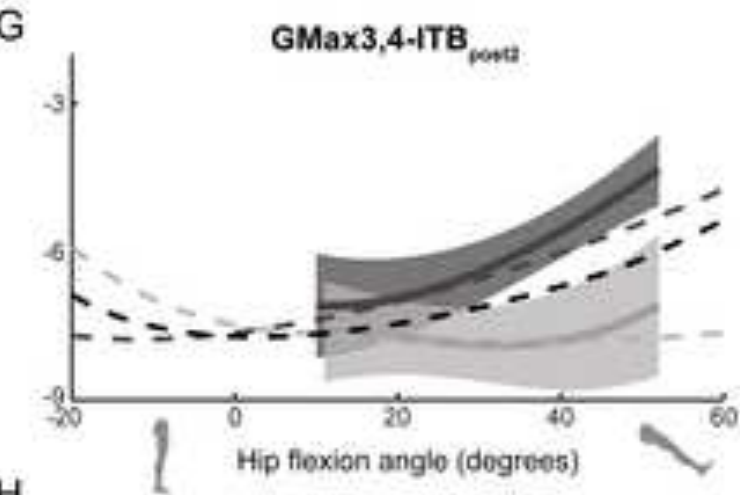

$\mathrm{H}$
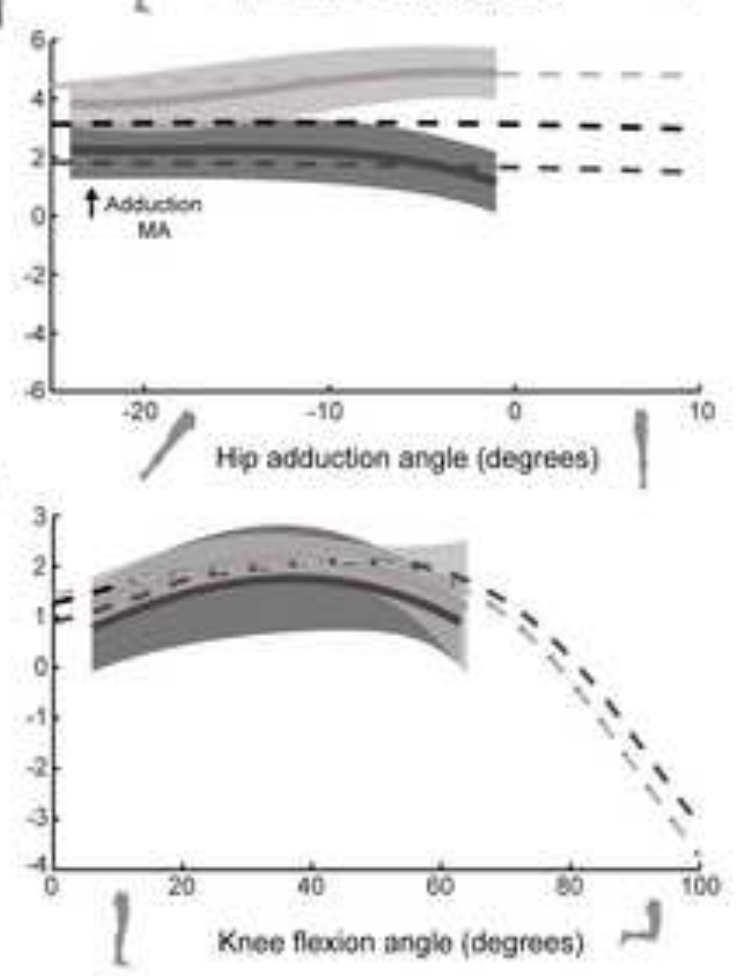


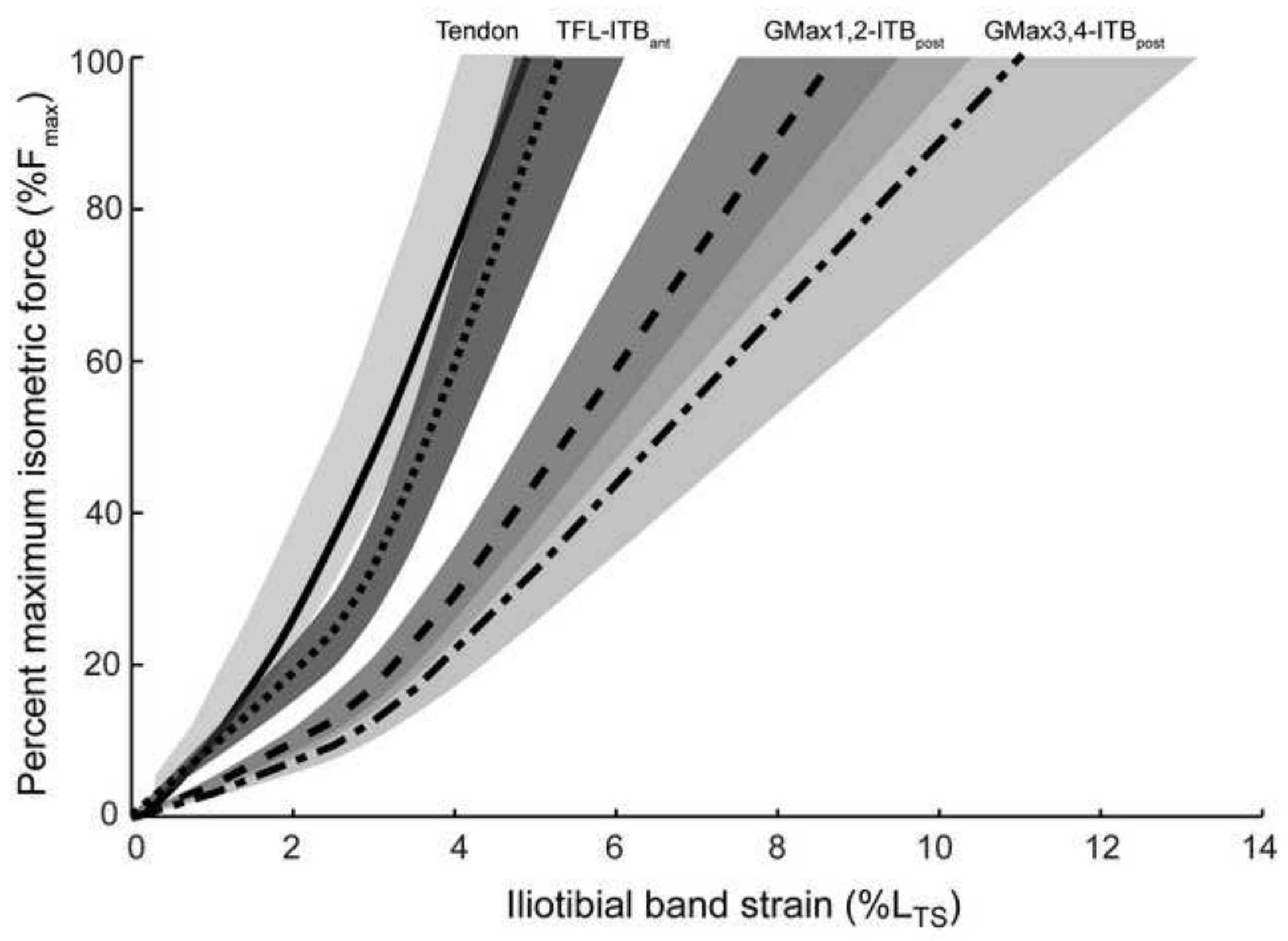




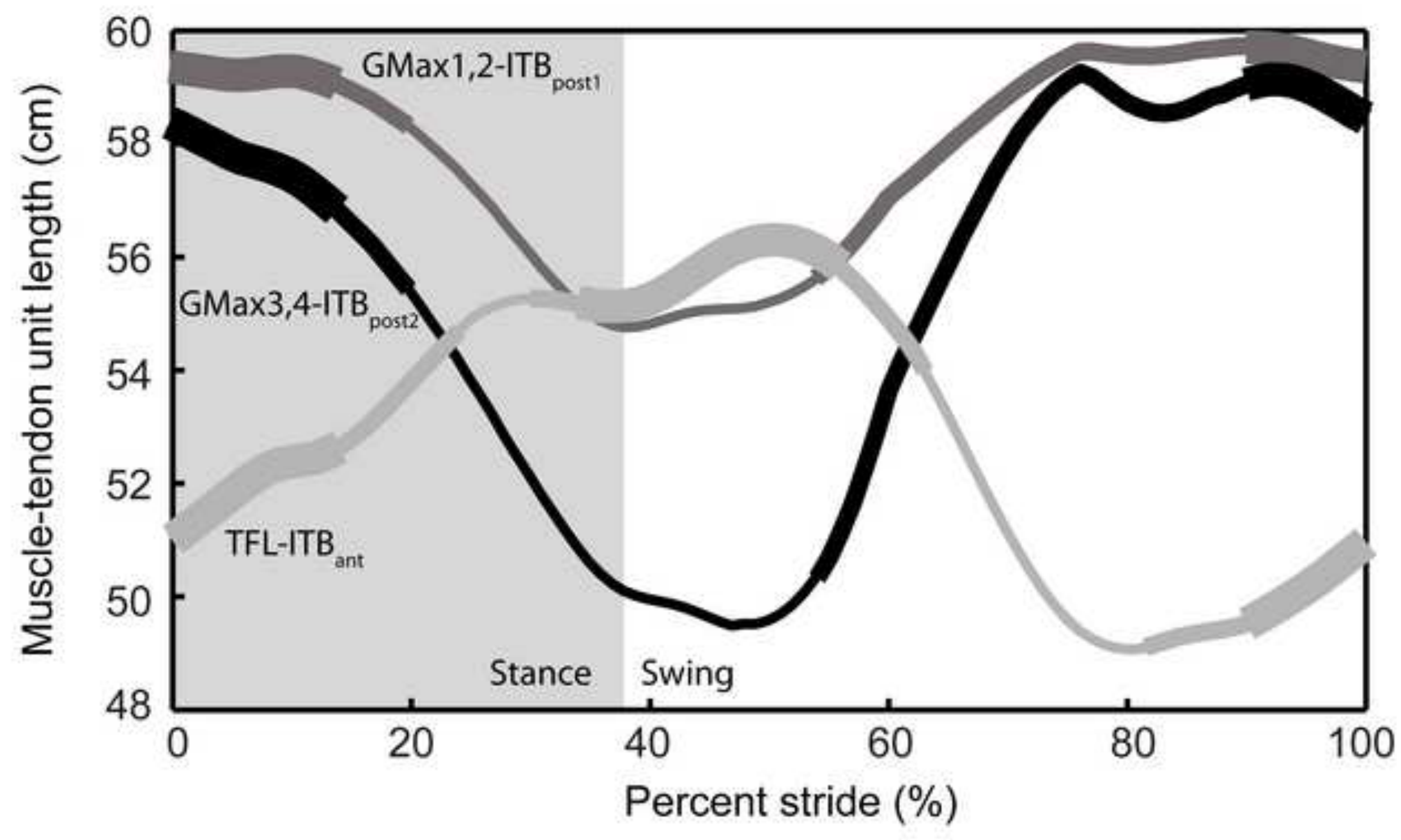


Figure 7

Click here to download high resolution image

A
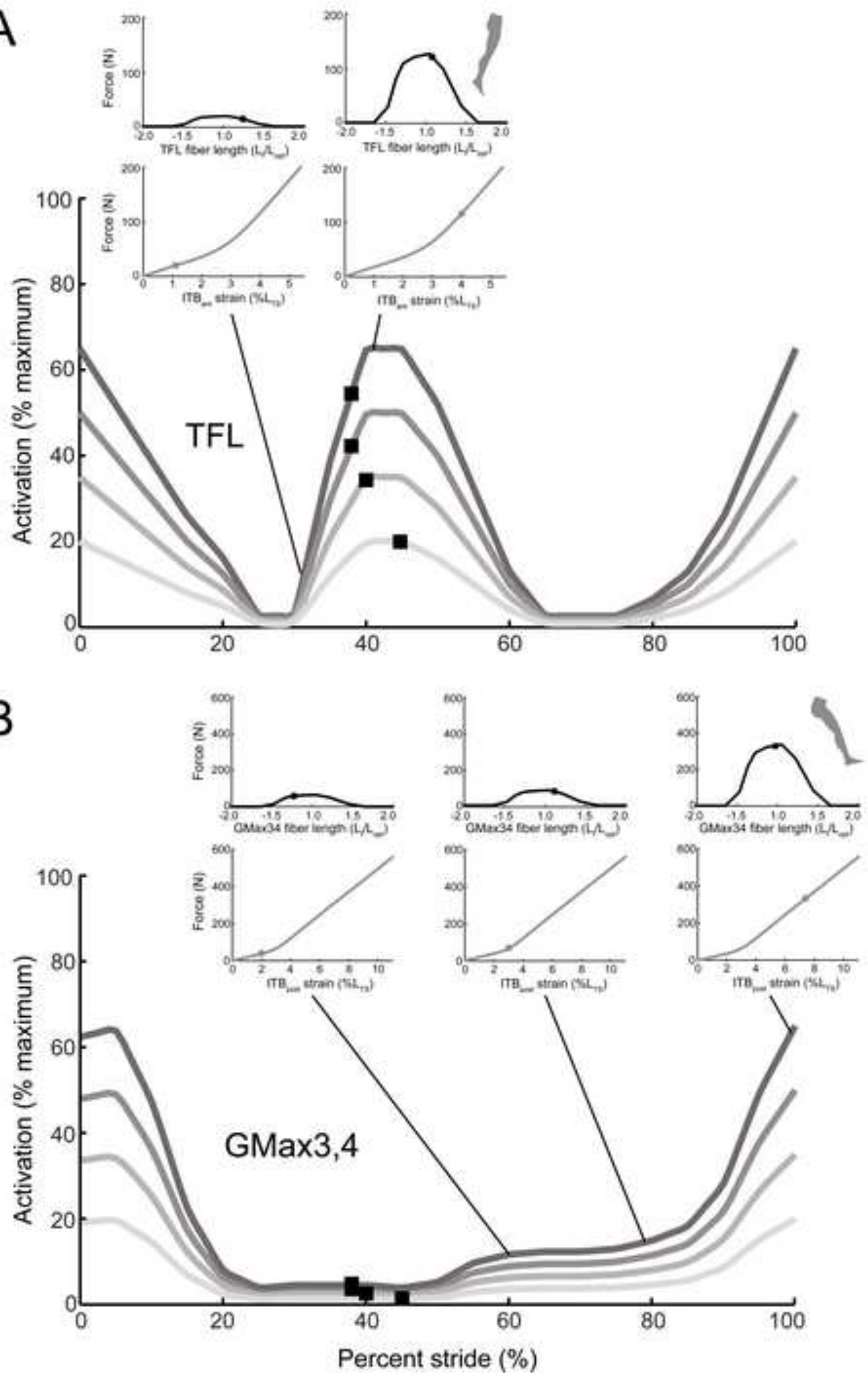

B 
Click here to download high resolution image

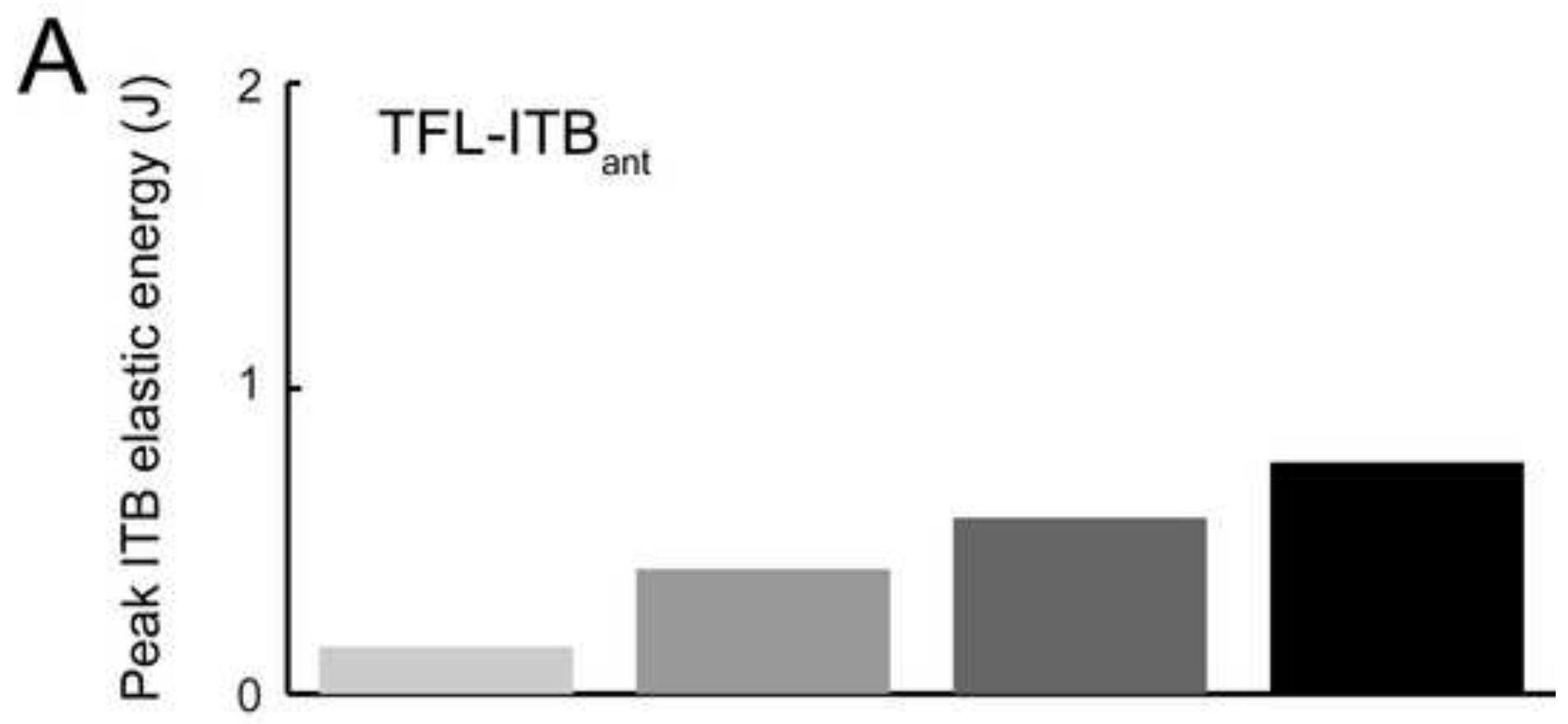

$\mathrm{B}$

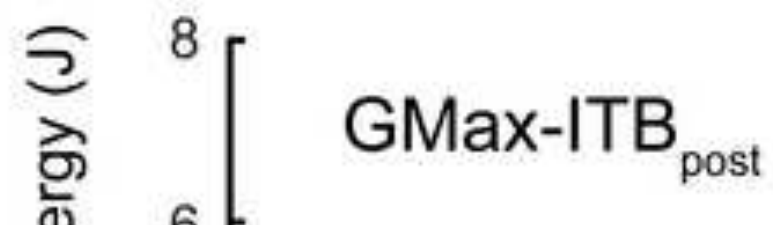

(1)

․․

$\frac{\pi}{0}$
$\frac{\pi}{0}$
$\frac{9}{-}$
$\frac{1}{\pi}$
0
0

4

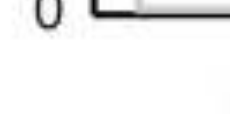

$2 \mathrm{~m} / \mathrm{s}$

$20 \%$

$3 \mathrm{~m} / \mathrm{s}$

$35 \%$

$4 \mathrm{~m} / \mathrm{s}$

$5 \mathrm{~m} / \mathrm{s}$

$65 \%$ 


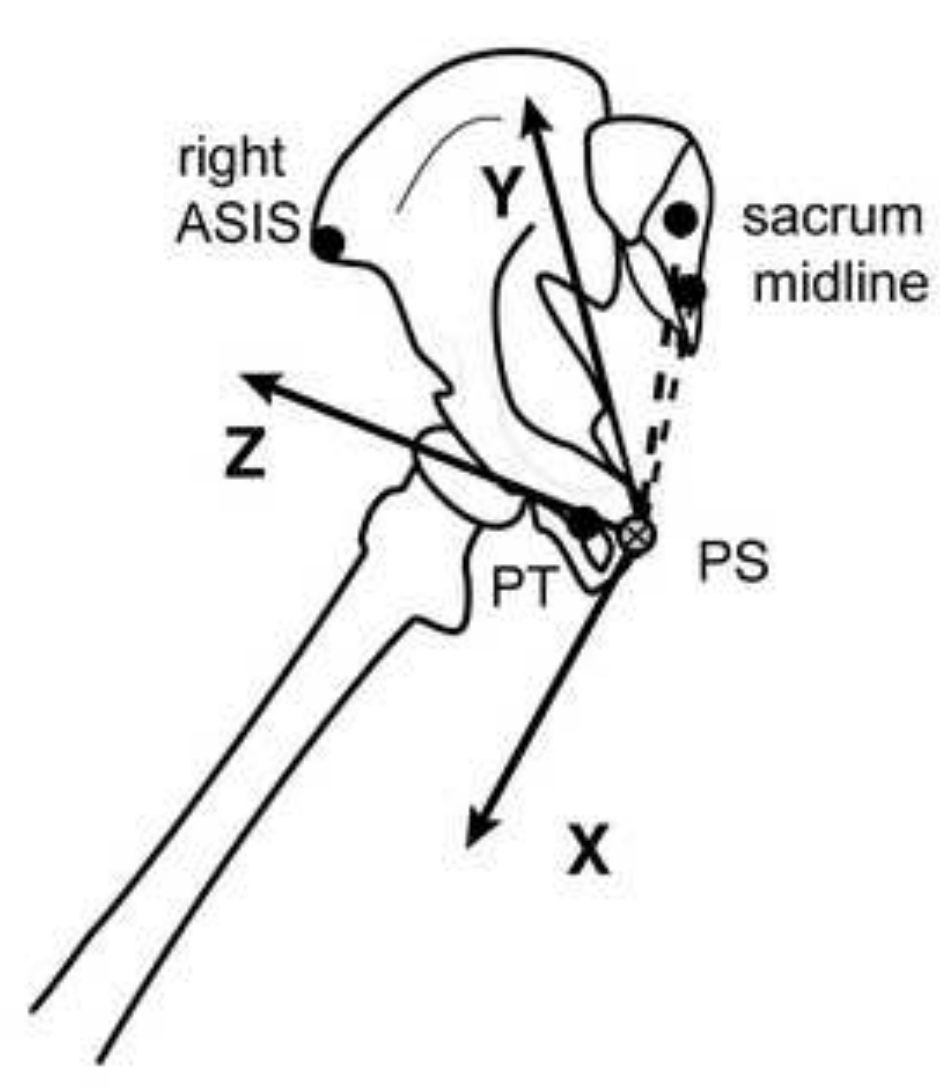

Pelvis Coordinate System

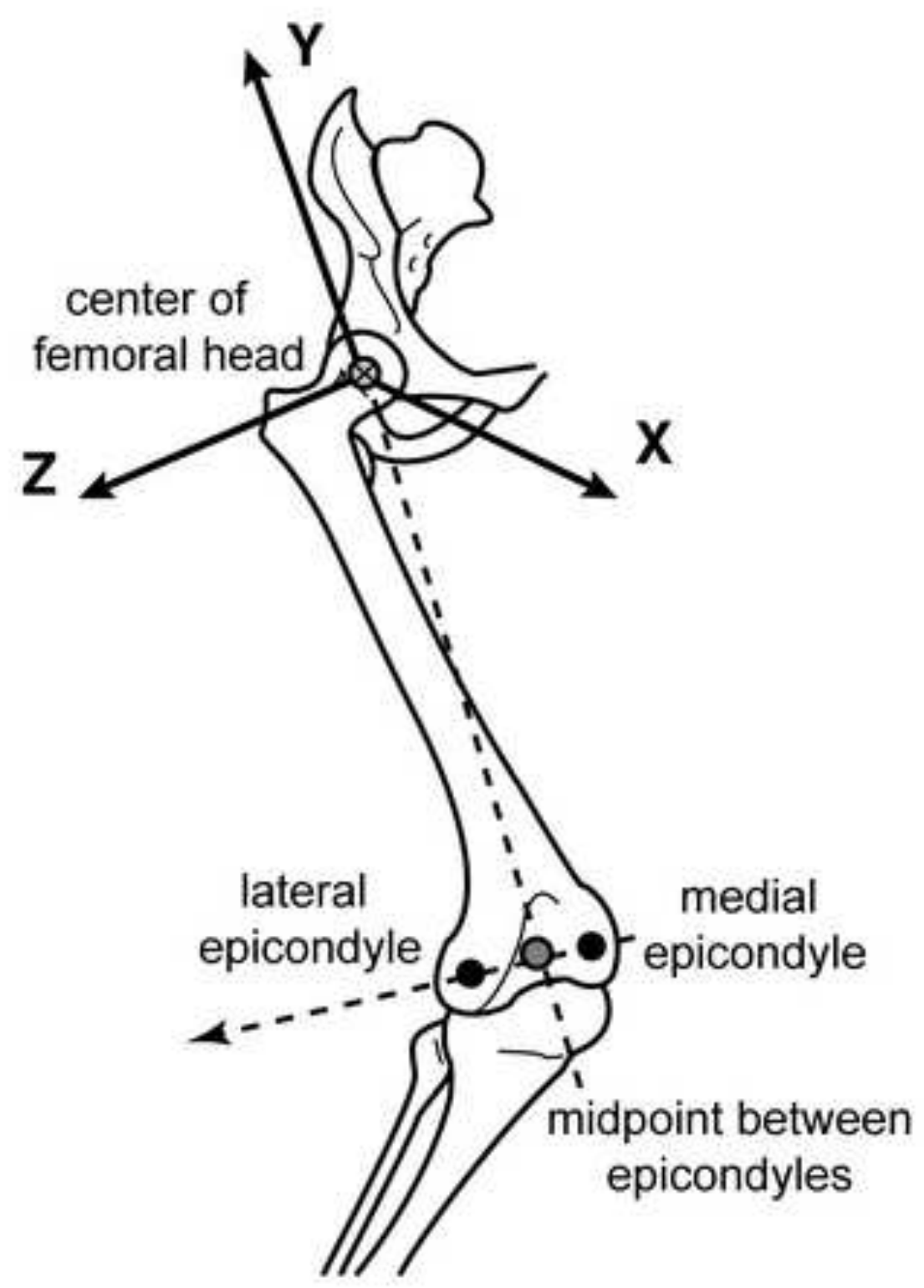

Femur Coordinate System 

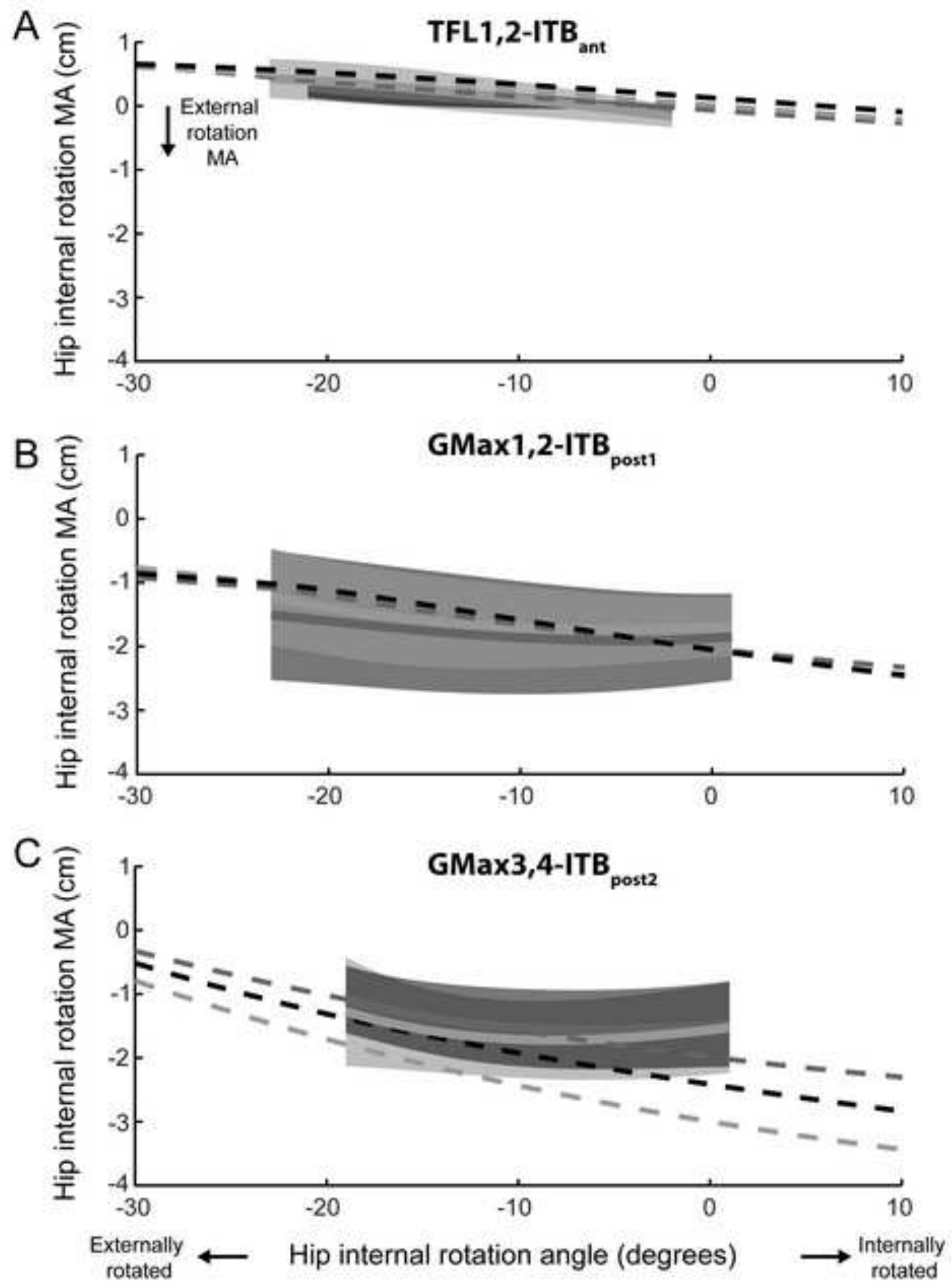
Table 1. Muscle architecture of tensor fascia lata (TFL) and gluteus maximus (GMax*)

\begin{tabular}{|c|c|c|c|c|}
\hline Muscle & $\begin{array}{l}\text { Mass } \\
(\mathrm{g})\end{array}$ & $\begin{array}{l}\text { Optimal fascicle length } \\
(\mathrm{cm})\end{array}$ & Pennation angle (deg.) & $\begin{array}{l}\operatorname{PCSA}^{\mp} \\
\left(\mathrm{cm}^{2}\right)\end{array}$ \\
\hline TFL & $35.5 \pm 9.6$ & $9.8 \pm 0.7$ & $1.1 \pm 1.1$ & $3.2 \pm 1.0$ \\
\hline GMax & $412.1 \pm 69.7$ & $14.4 \pm 0.7$ & $26.3 \pm 5.0$ & $30.6 \pm 5.1$ \\
\hline
\end{tabular}

${ }^{*}$ Pennation angle is not included in the PCSA calculation since our SIMM model multiplies PCSA, specific tension, and pennation angle to determine a muscle's maximum isometric force. 
Table 2. Muscle regional masses of tensor fascia lata (TFL) and gluteus maximus (GMax)

\begin{tabular}{ccc}
\hline Muscle & $\begin{array}{c}\text { Total mass of region }(\mathbf{g}) \\
(\mathrm{n}=5)\end{array}$ & $\begin{array}{c}\text { Percentage of mass inserting on ITB } \\
(\%) \\
(\mathrm{n}=2)\end{array}$ \\
\hline TFL1 $^{\infty}$ & $26.4 \pm 7.2$ & $100 \%$ \\
TFL2 & $21.4 \pm 5.5$ & $100 \%$ \\
GMax1 $^{¥}$ & $110.6 \pm 26.2$ & $44.6 \pm 4.9 \%$ \\
GMax2 $^{*}$ & $109.4 \pm 24.8$ & $52.7 \pm 7.8 \%$ \\
GMax3 & $121.9 \pm 19.8$ & $47.7 \pm 11.3 \%$ \\
GMax4 & $104.7 \pm 29.7$ & $71.7 \pm 28.3 \%$
\end{tabular}

Data from 5 adult males (mean age: $62 \pm 10$ years) are expressed as mean \pm s.e.m.

${ }^{\infty} \mathrm{TFL}$ was divided into two anterior-posterior regions based on origin and fascicle orientation.

${ }^{*}$ GMax was separated into four superior-inferior regions. 
Table S1. Parameters used to scale a generic Hill-type muscle model to TFL- and GMax-ITB muscle-tendon units

\begin{tabular}{ccccc}
\hline $\begin{array}{c}\text { Muscle-tendon } \\
\text { unit }\end{array}$ & $\begin{array}{c}\text { Maximum isometric } \\
\text { force }\left(\mathbf{N} ; \boldsymbol{F}_{\text {max }}\right)^{*}\end{array}$ & $\begin{array}{c}\text { Optimal fiber length } \\
\left(\mathbf{c m} ; \boldsymbol{L}_{\text {opt }}\right)\end{array}$ & $\begin{array}{c}\text { Pennation angle } \\
\text { (deg; } \boldsymbol{\theta})\end{array}$ & $\begin{array}{c}\text { Tendon slack length } \\
\left(\mathbf{c m} ; L_{T S}\right)\end{array}$ \\
\hline TFL1,2-ITB $^{\$}{ }^{\$}$ & 195.2 & 9.8 & 2.5 & 42.6 \\
GMax1,2-ITB $_{\text {post1 }}{ }^{*}$ & 455.9 & 15.2 & 26.3 & 42.3 \\
GMax3,4-ITB $_{\text {post2 }}$ & 558.6 & 16.7 & 26.3 & 41.0 \\
\hline
\end{tabular}

\$TFL PCSA, $L_{o p t}$, and $\theta$ and GMax PCSA and $\theta$ from our measurements.

* GMax $L_{\text {opt }}$ from Ward et al. (2009).

${ }^{*}$ Fmax calculated as the product of $P C S A$ and muscle specific tension of $61 \mathrm{~N} / \mathrm{cm}^{2}$ used by Arnold et al. (2010). 
Table S2. ITB thickness and width measurements used to calculate effective cross-sectional area and stiffness of each region

\begin{tabular}{|c|c|c|}
\hline ITB Region & Thickness (mm) & Width (mm) \\
\hline ITB $_{\text {ant }}$ & $0.87 \pm 0.34$ & $16.35 \pm 2.03$ \\
\hline ITB ${ }_{\text {post1 }}$ & $097+012$ & $16.85 \pm 1.85$ \\
\hline ITB post2 & $0.0-0.16$ & $15.33 \pm 1.86$ \\
\hline
\end{tabular}

